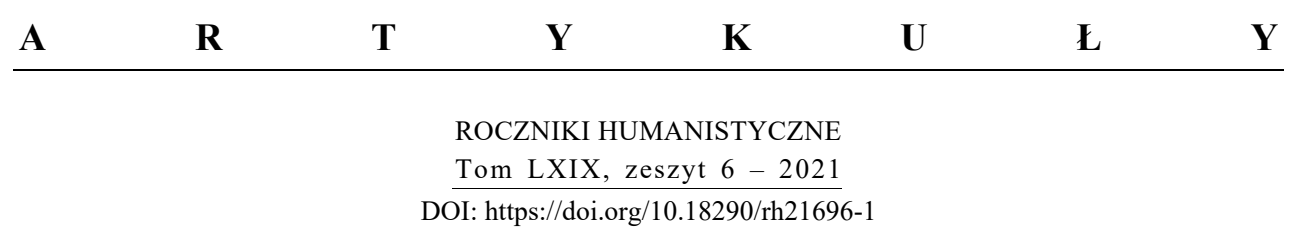

JANUSZ BIEŃ

\title{
RECURSOS LÉXICO-PRAGMÁTICOS EN LOS TITULARES DE LA PRENSA DEPORTIVA DIGITAL
}

\section{TITULARES DE PRENSA, FUNDAMENTOS TEÓRICOS}

Numerosos lingüistas de la tradición española se han dedicado al análisis del discurso periodístico ofreciendo descripciones de los encabezamientos de las noticias informativas. Nos referimos tanto a los trabajos de investigación lingüística como a los llamados libros de estilo, manuales de redacción de textos, elaborados por la tradición periodística española, sobre todo por grandes títulos nacionales como el País, El Mundo, La Vanguardia, El Marca u otros. En nuestros trabajos anteriores citamos varias definiciones de los titulares que no creemos necesario repetir aquí con detalles, y nos limitamos a algunas de ellas dedicando más espacio a aspectos prácticos. La definición de Alarcos Llorach parece ser la más dogmática en este ámbito ya que se trata de una de las primeras que se han propuesto en el marco de la lingüística española: «letreros con que se indica o se da a conocer el contenido, objeto o destino de un escrito impreso en los periódicos» (Alarcos Llorach, «El lenguaje de los titulares» 128). El mismo Alarcos Llorach, un lingüista muy prolífico, desarrolla en otros de sus trabajos varios aspectos que se esconden bajo esta propuesta rudimentaria. Así, por ejemplo, en Alarcos Llorach et al., Lengua española (171) hace referencia a «tres funciones fundamentales de los titulares: función distintiva, por la que se diferencia la noticia que encabezan de las

Dr hab. JANUSZ BIEŃ, prof. KUL - kierownik Katedry Językoznawstwa Romańskiego w Instytucie Językoznawstwa Katolickiego Uniwersytetu Lubelskiego Jana Pawła II; adres do korespondencji: Katolicki Uniwersytet Lubelski Jana Pawła II, Katedra Językoznawstwa Romańskiego, Al. Racławickie 14, 20-950 Lublin; e-mail: janusz.bien@kul.pl. ORCID: https://orcid.org/00000002-6134-593X. 
demás, la referencial, por la que indican brevemente y con claridad el contenido de la noticia y la expresiva, por la que despiertan el interés del lector» ${ }^{1}$. En general, las definiciones que aparecen en la literatura dedicada al asunto ponen acento tanto en la función sintetizadora de los encabezamientos como en su función expresiva. Esta última parece resaltarse por el enfoque que adopta Jorques (95-96): «la plasmación lingüística de la operación cognitiva de la atención, gracias a su colocación tipográfica en la noticia».

En general, los Libros de estilo aludidos antes, siguiendo los preceptos del buen periodismo, apuntan a la función informativa de los titulares afirmando que «constituyen el principal elemento de una información. Sirven para centrar la atención del lector e imponerle de su contenido» (El País 35) ${ }^{2}$. Grijelmo (456) desarrolla esta bifuncionalidad señalando que los titulares no solo sintetizan el contenido del texto que encabezan, sino que también jerarquizan la noticia en el mismo ejemplar y la confrontan con otras. La tradición polaca apunta también a la bifuncionalidad de los encabezamientos resaltando su función jerarquizadora: «[...] funkcję wartościującą. Wielkość nagłówka i liczba zajmowanych przez niego łamów orientują czytelnika w hierarchii ważności poszczególnych artykułów na kolumnie gazety» (Wolański 279). No cabe ninguna duda de que ambas funciones se interponen y parecen ser inseparables, frente a la constatación de numerosos especialistas de la prensa informativa según la cual los titulares de prensa «constituyen las únicas secuencias leídas por gran parte de consumidores diarios» (Nadal Palazón, «Verdades a medias»177).

\section{CUESTIONES TERMINOLÓGICAS}

En el lenguaje común suelen confundirse los significados de los vocablos título y titular que en los textos de lingüística adquieren sentidos distintos. No obstante, en el discurso periodístico, el término titular se refiere a un espacio tipográfico más amplio, siendo encabezamiento de la noticia, y título puede ser tan solo uno de sus constituyentes textuales. Gozalo Gómez (658) proporciona una explicación muy clara de ambos conceptos: «un titular suele tener la siguiente estructura: Antetítulo, TÍTULO, Subtítulo o sumario. El título,

\footnotetext{
${ }^{1}$ Citamos según Fuente González (161).

${ }^{2}$ Encontramos un enfoque parecido en El Mundo (39-40) y en Fuente González (173).
} 
también denominado cabeza ${ }^{3}$, es la parte principal de un titular. Su extensión no debe exceder de trece palabras y debe contener la información más importante, en el caso de las noticias, o la más llamativa en el caso de textos no puramente informativos».

Las opiniones de los especialistas de la prensa coinciden en que los elementos del titular diferentes al título (antetítulo, subtítulo) no son obligatorios y aparecen según el perfil del periódico, la importancia de la noticia o una actitud más o menos expresiva del periodista. Por otra parte, es obvio decir que un titular de extensión muy reducida queda indivisible y se identifica con el título.

A la hora de abordar los problemas terminológicos, conviene añadir que el término encabezamiento adopta un sentido todavía más general y tal vez funcione como hiperónimo frente a titular. No obstante, por motivos de simplificación, en el presente texto usamos el término titular tanto para designar el título (solo o con todos los elementos que lo desarrollan) como en un sentido general que se corresponde con cualquier tipo de encabezamiento de la noticia de prensa digital, gráficamente destacado. Huelga añadirse que, fuera del contexto lingüístico, tanto titular como título acuñan otros significados que no radican necesariamente en el ámbito periodístico, siendo este último mucho más abstracto y altamente polisémico.

Recordemos que, en polaco, el término mayor nagłówek designa aproximadamente cualquier titular aparecido en la prensa; tytut (título), podtytut (subtítulo), nadtytut (sobretítulo) o śródtytut (intertítulo) serían sus componentes de menor extensión textual. De hecho, en varias ocasiones el titular se reduce al título. Este enfoque lo encontramos, por ejemplo, en Wolański (278), que distingue claramente las partes del encabezamiento que acaban de enumerarse: «Tytuły w gazetach i czasopismach noszą nazwę nagłówków prasowych. Nagłówek prasowy składa się z wyróżnionego graficznie tytułu głównego $[\ldots]$ wraz $\mathrm{z}$ ewentualnym nadtytułem lub podtytułem. Pod względem ukształtowania edytorskiego nagłówki można podzielić na jednoczłonowe, które składają się tylko z tytułu głównego, i wieloczłonowe, które oprócz tytułu głównego mają nadtytuł i/lub podtytuł $[\ldots] \gg^{4}$.

Fuente González (173) constata que la existencia de varios componentes en un mismo titular es una contradicción tan solo aparente; no obstante, requiere del periodista una máxima concisión verbal. Según Nebot et al.

\footnotetext{
${ }^{3}$ Esta denominación sinonímica no parece tener el mismo alcance en lingüística española (cfr. Fuente González 177).

${ }^{4}$ El resalte es nuestro.
} 
(298) el hecho de recurrir a los antetítulos o subtítulos radica en la necesidad de repartir y especificar la información principal de la noticia que no cabe en un título simple: «[...] el título en el que se centra el contenido principal que se quiere destacar; los antetítulos que suelen precisar el alcance de los títulos; subtítulos y sumarios que especifican determinados aspectos o puntos, aunque a veces cumplen la misma función que el antetítulo» ${ }^{5}$.

\section{TIPOS DE TITULARES}

Los recursos formales empleados con más frecuencia combinados con funciones dominantes llevan a los lingüistas a proponer algunas tipologías de los titulares de prensa. Estas, casi siempre se reducen a una bipartición establecida en función de algún rasgo formal o discursivo mayor. El parámetro básico de la división es, desde luego, la extensión textual de los titulares o el grado de la condensación del contenido. Otras clasificaciones combinan criterios típicamente textuales con los semántico-comunicativos y discursivos como fuerza enunciativa de los títulos, actitud del autor frente a los hechos relatados o evidencias. A continuación presentamos algunas de estas biparticiones más destacadas que se proponen en el marco de la lingüística española:

- titulares amplios y concentrados (según la extensión textual y condensación del contenido)

- explícitos e implícitos (según la intencionalidad del autor)

- objetivos y comprometidos (según la actitud del periodista) ${ }^{6}$

- titulares-motor y titulares-focos, titulares-rótulo o titulares enunciativos (según la carga semántica y la fuerza comunicativa de los enunciados. ${ }^{7}$

En la parte práctica del presente trabajo nos interesa particularmente la última propuesta de clasificación ya que el criterio según el cual se establece, marca tal vez una diferencia esencial entre la prensa seria que se caracteriza por el rigor informativo y la prensa con un perfil más bien sensacionalista que recurre a distintas omisiones en los títulos con el fin de focalizar la atención de los lectores en un aspecto determinado:

Descanso: debilidad blanca (as.com; 21.10.2020)

Solomon marca el 0-3 del Shakhtar ante el Real Madrid (md.com; idem)

Szok w Madrycie! Real stracił trzy gole w I połowie! (sport.pl; idem)

\footnotetext{
${ }^{5}$ Citado en Fuente González (173).

${ }^{6}$ Cfr. Oliva Marañón (62-63)

${ }^{7}$ Véanse Gozalo Gómez (659) y El Mundo (40).
} 
El primer titular está privado de contenido gramatical y funciona como señalador enunciativo, como un foco en la noticia; por ello posee más fuerza comunicativa que los otros dos titulares en los que, mediante el verbo y sus complementos, se proporciona una información plena acerca de lo ocurrido.

\section{RECURSOS FORMALES}

Por motivos mencionados más arriba, los autores de los titulares de prensa intentan expresar lo máximo sirviéndose de todo tipo de recursos que permiten, por un lado, resumir la noticia y, por otro, alcanzar algunos efectos pragmáticos para provocar o animar al lector.

Nadal Palazón («Rasgos formales...»174, 189 y ss.) divide los rasgos formales de los titulares de prensa en dos clases: constantes y variables. A los primeros pertenecen bimembraciones de los enunciados: elipsis, recursos nominales y presente histórico; entre los segundos, el autor mejicano clasifica: tercera persona impersonal, verbo inicial, el llamado potencial citativo o criptónimos. No pretendemos desarrollar aquí esta interesante propuesta de clasificación de los recursos, dados el marco reducido del presente texto y el enfoque que adoptamos. Proponemos dividir los recursos en algunas clases rudimentarias según el plano lingüístico en el que son analizables: a) gráficos b) léxicos c) morfo-sintácticos b) retóricos o pragmáticos.

Los periódicos digitales, muy flexibles frente a las variaciones gráficas que se presentan en la lengua española, no condenan su uso, visto que estas desempeñan una función expresiva muy importante. He aquí un par de ejemplos que hablan por sí solos (el resalte es nuestro):

Un tropiezo reLLevante (marca.es; 04.05.14)

Marcó Asensio (as.com; 22.09.18)

¡BarVARidad! (sport.es; 23.09.18)

Para la prensa digital, el plano léxico constituye una fuente de experimentos casi inagotable y los periodistas recurren con voluntad a lexemas con valor expresivo, a veces hasta vulgarismos, préstamos o neologismos, lo permita o no el carácter de la prensa:

Político desengañado, empresario fulgurante (elmundo.es 30.04.14)

En mi puta vida he cobrado un sobre (idem)

«Biciosos», respuestas a las preguntas que surgen al desplazarse en bicicleta (yahoo.es; 01.05.14) 
A nuestro juicio, los recursos formales son los más relevantes para la redacción de los titulares. Tenemos en cuenta aquí los mecanismos que permiten omisiones argumentales, temporales y deícticas: elipsis, sea del sujeto complemento o verbo, o estructuras impersonales ${ }^{8}$ :

Un cadáver contra Gerry (elmundo.es; 01.05.14)

Una debacle con calmante en Berlín (idem)

México: encuentran 5 cadáveres con tiro de gracia (Hoy; 20.01.10)

HAITÍ: VAN 70 MIL ENTERRADOS (El Universal 18.01.10) ${ }^{9}$

Los recursos retóricos se reducen al uso de distintas figuras, que requieren un saber teórico por parte del periodista; por ello, su frecuencia no puede compararse con la de los recursos gramaticales:

El alcohol en polvo, cacao legislativo (metáfora; elmundo.es; 30.04.14)

Afectuosamente Mou (frase hecha; idem)

Las nominalizaciones $\mathrm{u}$ formas nominales funcionalmente asemejadas permiten elidir tanto el verbo como los argumentos ${ }^{10} \mathrm{y}$ por esta razón son, tal vez, los recursos más frecuentes en cualquier tipo de encabezamiento:

Entre penas y penaltis (as.com; 8.11.2020)

El Clásico resucita al Madrid (marca.com; 24.10.2020)

En la prensa digital seria a la que pertenecen, según nuestro enfoque, tanto las páginas web de los medios de comunicación como cadenas informativas de radio o televisión, censamos, en general, los titulares con información argumental y temporal plena, que no generan ningún efecto discursivo indeseado:

Detenido el joven que lanzó un plátano a Dani Alves (rtve.es; 30.04.14)

Muere el actor británico Bob Hoskins a los 71 años (idem).

\footnotetext{
${ }^{8}$ Es interesante leer otra constatación de Nadal Palazón («Rasgos formales» 189) sobre una variación dialéctica de la aparición de la tercera persona impersonal en los titulares de prensa: «Los periódicos hispanoamericanos presentan una indudable tendencia al uso de construcciones de interpretación impersonal en tercera persona del plural. El fenómeno es mucho menos frecuente en la prensa española, pero no imposible de rastrear. Hay que insistir en que, en los diarios de Hispanoamérica, prácticamente no hay edición que no contenga una buena cantidad de ejemplos, ya sea que se conozca o no el agente del proceso».

${ }^{9}$ Ambos ejemplos según Nadal Palazón («Rasgos formales» 178).

${ }^{10}$ Nos informan Hooper and Thompson (285) que tan solo 5\% de las nominalizaciones (material inglés) conllevan la información argumental plena (sujeto y dos complementos).
} 


\section{ESTATUS DE LA PRENSA DIGITAL}

Un desarrollo constante de los medios que se observa desde hace décadas lleva a los lingüistas a considerar la prensa digital como un tipo de lenguaje independiente, que difiere tipológicamente de la prensa tradicional. En nuestra opinión, algunos subtipos de prensa electrónica han de distinguirse según la manera de abordar los contenidos o según algunos de sus parámetros técnicos. Siguiendo este primer enfoque, podemos agrupar los portales que representan la prensa digital en las categorías siguientes:

- la prensa informativa diaria (versiones electrónicas de los periódicos diarios existentes en versión tradicional o portales informativos)

- títulos que representan a los medios no escritos (páginas web de las emisoras de radio o cadenas de televisión)

- páginas que reúnen lo informativo con el ocio y distracción

Por extensión de la palabra y por motivos prácticos y funcionales, todos los tipos de portales digitales que acabamos de listar se consideran como prensa digital informativa y como un género discursivo independiente. A nuestro juicio, es mucho más indicado proceder así, cuando el análisis atañe solamente a los encabezamientos y no al cuerpo del texto donde se recogen más diferencias estilísticas o ideológicas entre distintos medios digitales.

El parámetro técnico evocado más arriba permite establecer los subtipos de prensa digital según el soporte electrónico al que se destinan: teléfono móvil, tableta $\mathrm{u}$ ordenador ${ }^{11}$. Como bien es sabido, la disposición tipográfica del texto publicado en una página web clásica no siempre se corresponde con su versión destinada a los tamaños menores que requieren más concisión formal y un uso limitado de recursos hiper- y paratextuales (fotos, comentarios, etc.).

Hoy en día, los medios de comunicación masiva disponen de una tecnología muy avanzada; por ejemplo, conexión por satélite que permite una transmisión de información instantánea. Nada impide al autor modificar encabezamientos o textos en formato digital, según si le llegan nuevas informaciones sobre el acontecimiento comentado. Este fenómeno se observa, sobre todo, en noticias de primer plano que provocan a los lectores o impactan al mundo. Para ilustrar esta idea citamos a continuación un titular que sufre un cambio esencial en el

\footnotetext{
${ }^{11}$ Según Estudio Anual de Medios de Comunicación 2017 de la IAB elaborado por Elogia «El ordenador (que destaca entre el target senior) y el móvil (que sobresale entre los más jóvenes) son los dispositivos de conexión más habituales. La Tablet es más habitual a partir de los 31 años».

Estudio Anual de Medios de Comunicación 2017, blog.elogia.net/estudio-anual-de-medios-decomunicaci\%C3\%B3n-2017-iab. Accessed 25 Oct. 2020.
} 
periodo de una hora (nuevos aspectos de la noticia salen a luz) y cobra una forma definitiva en que la información argumental se ve reducida por la decisión del autor, seguramente mucho más consciente de lo ocurrido una hora después de la aparición del primer titular. La versión polaca que consultamos en calidad de tertium comparationis equivale en el plano informativo (reducción argumental) a la primera noticia de marca.com:

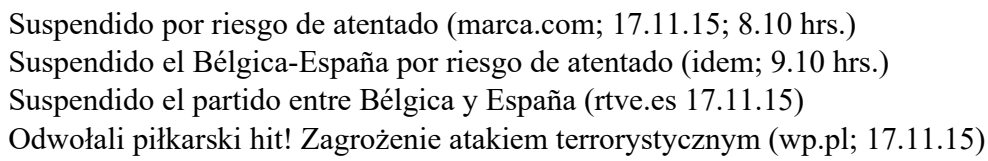

En el primer ejemplo de la serie, para aclarar la noticia, el autor recurre a efectos hipertextuales, ya que el enunciado va acompañado de una foto con los jugadores de la Roja. Se trata, sin duda, de aumentar la función expresiva y apelativa del titular que sugiere fuertemente que la selección española fuera involucrada en el evento aludido; el lector se queda confundido.

No cabe duda de que los elementos hipertextuales o extralingüísticos son empleados con más frecuencia en la prensa deportiva o prensa de sensación que en la prensa informativa nacional. Cualquier recurso de este tipo puede funcionar como estratagema periodística al crear un contexto indeterminado o ambiguo para los lectores. En algunas ocasiones, el hipertexto funciona como transmisor del verdadero contenido del titular. Esto ocurre cuando este se reduce a elementos expresivos o frases hechas sin referencia externa:

Pourquoi? Warum? Why? (rtve.es; 16.11.15)

(con velas encendidas dos días después del atentado; un subtítulo de abajo lo explica todo)

El titular que sigue representa un tipo enunciativo extremo en el que las supuestas reacciones del mundo frente a la noticia son más importantes que el contenido mismo que se reduce a un solo hecho, muerte de la estrella del fútbol. De esta manera, por un lado, los efectos hipertextuales, los contenidos de los llamados lead o antetítulos adquieren más fuerza expresiva; por otro lado, el autor puede aportar modificaciones significativas en un lapso de tiempo muy breve:

Argentyńskie media: Nie żyje Diego Maradona (wp.pl; 25.11.2020; 17.40 hrs.)

PILNE (antetítulo) Diego Maradona nie żyje. Lekarzom nie udało się go uratować (wp.pl; idem; 17.48 hrs.)

Fallece Maradona (marca.com; 25.11.2020; 17.40 hrs.) 
Fallece la leyenda del fútbol a causa de un paro respiratorio (idem; $23.00 \mathrm{hrs}$.) Hasta siempre, Diego (antetítulo) Muere Maradona (as.com; 25.11.2020; 17.40 hrs.) Diego que estás en los cielos (idem; 23.00 hrs.)

\section{CORPUS Y METODOLOGÍA DEL CENSO DE LOS DATOS}

El corpus de referencia del que nos servimos en el presente trabajo consta de algunas partes independientes. El corpus I, mero objeto de nuestro análisis práctico, contiene una serie de titulares recogidos en cinco fuentes digitales seleccionadas: dos madrileñas, dos catalanas y una polaca. Con vistas a contrastar la semántica de los enunciados y los recursos empleados en los titulares, hemos optado por los medios que se adscriben a dos campos rivales. Aunque se trata de analizar la prensa deportiva, las tensiones sociales, políticas o ideológicas entre Madrid y Barcelona llevan a creer que las supuestas diferencias no se limitarán al plano puramente futbolístico. Así pues, hemos decidido rastrear los títulos de prensa nacional más grandes: dos periódicos digitales madrileños: as.com y marca.com, dos periódicos digitales que tienen su sede en Cataluña sport.es y mundodeportivo.com y el portal deportivo polaco sport.pl en calidad de tertium comparationis - al estar fuera de cualquier conflicto ideológico, deportivo o social que diferencie ambas comunidades de la Península Ibérica esperamos que publicase titulares objetivos desde el punto de vista léxico o pragmático.

La primera parte de este subcorpus es de tipo continuo en la medida en que se han recogido todos los titulares que hablan de las actuaciones deportivas del Real Madrid y del Fútbol Club Barcelona (en total 160) en un periodo de tiempo determinado (entre 2.02.2018 y 13.05.2020). La segunda parte es una ampliación y consta de 160 nuevos ejemplos, recientemente recogidos en las mismas fuentes (entre 1.10.2020 y 20.12.2020) y tiene un carácter discontinuo. Por supuesto no se trata de titulares seleccionados de manera premeditada, sino de los que relatan hechos importantes y desde la perspectiva futbolística manifiestan un valor diacrítico para el presente estudio. Esta parte de nuestra base de ejemplos sigue estrictamente todas las pautas teóricas indicadas por los especialistas de la lingüística del corpus. Recordemos brevemente que se trata de los parámetros de índole científica como fecha de publicación semejante, una misma temática, un mismo género lingüístico o literario, un estilo semejante o transmisión por un mismo medio tradicional 
o digital ${ }^{12}$. El segundo subcorpus es de tipo mixto, no continuo y consta de un centenar de ejemplos recogidos en algunos títulos digitales españoles y polacos (v. la bibliografía) que nos sirven para ilustrar algunas pautas teóricas o algunas tendencias de la prensa digital española y catalana.

\section{ANÁLISIS DE LOS DATOS EMPÍRICOS}

En el presente apartado nos proponemos llevar a cabo un análisis práctico de los titulares censados en el corpus en función de algunos parámetros funcionales y discursivos. Con el propósito de determinar qué rasgos manifiestan los titulares deportivos catalanes, españoles (y polacos) hemos considerado necesario dividirlos en dos grupos: los que comentan las actuaciones del Real Madrid y los que relatan los eventos protagonizados por el Fútbol Club Barcelona. En el paso siguiente, hemos llevado a cabo un análisis cuantitativo y cualitativo contando las ocurrencias siguientes: (1) titulares publicados por los periódicos citados según relaten los hechos de manera neutra, positiva o negativa, (2) número de titulares motor (factual) y titulares-foco (enunciativos) en cada fuente y (3) tipo de recursos más frecuentes en cada fuente del corpus.

La primera parte de nuestra investigación práctica confirma y resalta las tendencias que se propusieron tras el rastreo de la mitad de los ejemplos (subcorpus I; Bień, «Observaciones...» 159). Como mencionamos en el trabajo citado, un hecho que se observa de manera constante es la falta de equivalencia semántica entre los titulares de la prensa catalana y madrileña en la medida en que es muy difícil encontrar el caso en que todos los titulares aborden un mismo evento según el mismo enfoque semántico. Siguiendo esta pista, hemos notado que no resulta muy fácil encontrar una valoración positiva de la actuación exitosa del Real Madrid en todos los ejemplos de una misma serie, pero sí lo es cuando el evento está protagonizado por el Fútbol Club Barcelona, como en estos ejemplos en que los medios madrileños y los catalanes emplean curiosamente un mismo léxico valorativo:

El Barça se sube a la liga (as.com; 16.12.2020)

Un Barça de dos caras tumba al líder (marca.com; idem)

El Barça tumba al líder y se agarra a la liga (sport.es; idem)

El mejor Barça tumba a la Real (md.com; idem)

\footnotetext{
${ }^{12}$ Entre varios estudios que abordan estas cuestiones, véase Lewandowska-Tomaszczyk (52 y ss.).
} 
Odrodzenie Barcelony? Pokonała dotychczasowego lidera ligii hiszpańskiej! (sport.pl; idem)

En cambio, cuando se trata de una derrota, que siempre es motivo de críticas en la prensa deportiva, una valoración negativa de la actuación de cualquier campo es natural y muy frecuente en todos los medios que se investigan en el presente trabajo:

Cristiano ejecuta al Barça (as.com; 8.12.2020)

Cristiano hunde más al Barça (marca.com; idem)

El Barça sigue a la deriva y se complica mucho la Champions (sport.es; idem)

El Barça naufraga también en Europa (md.com; idem)

Juventus zbił Barcelonę na Camp Nou (sport.pl; idem)

Un mecanismo que se observa con más frecuencia en los titulares publicados por los periódicos catalanes y que sirve para menospreciar los éxitos del rival consiste en canalizar la atención del lector en los hechos del segundo plano a costa de lo realmente factual. En tal caso, sucesos y protagonistas alejados del centro de la noticia cobran más importancia en detrimento del hecho principal que, en este caso, es el éxito madridista:

Benzema, bonito del norte (as.com; 20.12.2020)

Benzema decide un partidazo (marca.com; idem)

El Real Madrid sufre para ganar al Eibar (md.com; idem)

El VAR se salta unas manos de Sergio Ramos (sport.es; idem)

El Madrid está aquí (as.com; 12.12.2020)

Un gran Madrid reabre LaLiga (marca.com; idem)

El Real Madrid gana al peor Atlético (md.com; idem)

El Real Madrid aprieta la liga por incomparecencia del Atlético (sport.es; idem)

Real Madryt pewnie pokonał faworyta Zinedine'a Zidane'a do wygrania ligi

(sport.pl; idem)

Una observación minuciosa de los 320 titulares recogidos en nuestro corpus de referencia entre los cuales aparecieron 128 en los medios madrileños (as.com y marca.com), 128 en los periódicos catalanes (sport.es y mundodeportivo.com) y los 64 restantes en el portal polaco sport.pl, llegamos a observar las siguientes tendencias:

1. Los medios catalanes, en mayor medida sport.es que mundodeportivo.com, amortiguan los éxitos del campo rival con falta de valoración o con enunciados 
neutros $^{13}$. Los medios madrileños casi siempre valoran positivamente los éxitos del club rival.

2. En los medios catalanes son más frecuentes los titulares de tipo motor, que constan de varios elementos y proporcionan a menudo una información gramatical plena; los portales madrileños manifiestan cierta predilección por titulares de tipo enunciativo, cortos y expresivos, a menudo privados de información gramatical.

3. Los recursos que se emplean en todos los medios analizados aquí son de doble naturaleza: léxica y formal; entre los primeros dominan adjetivos valorativos negativos o positivos y, además, se nota una predilección de la prensa madrileña por frases hechas con sentidos figurados o cualquier tipo de expresiones cortas enunciativas, menos presentes en los medios catalanes. Entre los procedimientos sintácticos, el más frecuente en los titulares es la elipsis del verbo, normalmente superfluo, visto un contexto muy preciso. La elipsis del sujeto no se da por motivos comunicativos obvios: siempre constituye el tema de la noticia.

Varios de estos fenómenos se explican tal vez por el fenómeno que algunos especialistas llaman infoentretenimiento que en la prensa deportiva supera a la vocación informativa. Es más, algunos hablan hasta de un cierto menosprecio que brindan a los periodistas deportivos sus compañeros de las redacciones típicamente informativas: "Consideramos que una de las causas de esta minusvaloración puede estar motivada por uno de los aspectos que señala el autor, que una parte notoria de la información se dedica a cubrir diversos pormenores, no necesariamente deportivos, de la vida del deportista famoso» (Gómez Bueno 271).

\section{COMENTARIOS Y CONCLUSIONES}

Tras este breve repaso de los titulares de la prensa digital, nos damos cuenta de que su análisis en términos objetivo/comprometido, serio/sensacionalista, enunciativo/no enunciativo está relacionado con la actitud de la prensa $\mathrm{y}$, en general, con el carácter de los medios de comunicación en comunidades que viven tensiones o conflictos de cualquier índole. Como los titulares son

\footnotetext{
${ }^{13}$ No pretendemos entrar aquí en el debate acerca del perfil ideológico o social de la prensa deportiva catalana y castellana. A este respecto es interesante leer algunas opiniones que van más allá y apuntan a la transgresión de las normas éticas del periodismo por parte de los medios deportivos catalanes (véase el párrafo «La intimidad y el honor de los actores de las noticias en la prensa deportiva» en Gómez Bueno (268 y ss.)).
} 
textos muy breves y requieren por parte del autor una máxima condensación del contenido, lo que genera efectos como vaguedad o indeterminación del contenido, que puede desembocar, por consiguiente, en una manipulación del lector. En este corpus temático los periódicos deportivos catalanes, por motivos que no resultan obvios, parecen sacar más provecho de dichos mecanismos, se convierten en medios más comprometidos y transgreden, en cierta medida, la frontera entre el juego discursivo y la manipulación. Los medios madrileños, por un lado, no prescinden de hablar positivamente de los éxitos del club contrario, tienden a emplear enunciados cortos y enunciativos que, por otro lado, están muy elaborados y son difícilmente analizables como comprometidos, manipuladores o sensacionalistas. Finalmente, el portal polaco guarda, en la mayoría de los casos, una actitud no comprometida, empleando recursos positivos o negativos según la necesidad discursiva, publicando titulares extensos, centrados en el aspecto informativo.

Una tendencia al sensacionalismo más pronunciada por parte de los medios catalanes analizados aquí puede estar relacionada con los conceptos generales que los teóricos adscriben a la prensa deportiva que invita al lector a participar en el evento relatado como atractivo o dramático. A lo largo del presente texto hemos ido señalando que en este tipo de prensa, lo informativo cede la prioridad a lo espectacular ${ }^{14}$. No obstante, no estamos sentenciando que esta tendencia sea constante en el seno de toda la prensa deportiva; para afirmarlo, deberían investigarse otros campos semánticos, una tarea que queda pendiente.

\section{BIBLIOGRAFÍA}

Alarcos Llorach, Emilio. «El lenguaje de los titulares». Lenguaje en periodismo escrito, edición a cargo de Lázaro Carreter, Juan March, 1977, págs. 127-147.

Alarcos Llorach, Emilio, et al. Lengua española. Santillana, 1989.

Bień, Janusz. «Estratagemas discursivas en los titulares de la prensa deportiva española catalana y polaca». Nuevos estudios de lingüistica románica/Nouvelles études de linguistique romane, edición a cargo de Janusz Bień y Tomasz Niestorowicz, Werset, 2018, págs. 11-25.

Bień, Janusz. «Observaciones sobre los titulares en la prensa deportiva digital». Voces dialogantes. Estudios en homenaje al profesor Wiaczestaw Nowikow, edición a cargo de Antonio María López González, et al. Wydawnictwo Uniwersytetu Łódzkiego, 2019, págs. 153-162.

El Mundo. Libro de estilo del diario 'el Mundo'. Temas de hoy, 2002.

${ }^{14}$ «Pero, si bien es cierto que el periodismo deportivo suele atraer audiencias con intereses muy específicos (el resultado y los pormenores del partido de fútbol, las expectativas que tiene un boxeador de cara a su adversario...), puede afirmarse que su relevancia en términos de información periodística se extiende al valor universal de lo espectacular» (Domínguez Pérez 6), (la numeración de páginas es nuestra). 
El País. Libro de estilo. Aguilar, 2014.

Domínguez Pérez, Jaime Pablo. «Ensayo sobre la cuádruple espectacularidad del periodismo deportivo». Razón y Palabra, vol. 69, 2009. dialnet.unirioja.es/ejemplar/234651. Accessed 17 Sept. 2018.

Fuente González, Miguel Ángel. «Introducción a los titulares de la prensa». Tabanque, vol. 7, 1991, págs. 159-81.

Gómez Bueno, Javier. «Análisis ético de la prensa deportiva escrita en Cataluña, en el empleo de técnicas sensacionalistas». Razón y Palabra, vol. 85, 2014, págs. 256-280. www.revistarazonypalabra.org/ index.php/ryp/article/view/429. Accessed 16 Sept. 2018.

Gozalo Gómez, Paula. «Los titulares de prensa en el aula de español lengua extranjera. Propuestas didácticas». Medios de comunicación y enseñanza del español como lengua extranjera, edición a cargo de Hermógenes Perdiguero and Antonio Álvarez, Universidad de Burgos, 2004, págs. 654-665.

Grijelmo, Ángel. El estilo del periodista. Taurus, 2001.

Hopper, Paul, and Sandra Thompson. «Transitivity in grammar and discourse», Language, vol. 56, 1980, págs. 251-299.

Jorques, Daniel. Discurso e información. Estructura de la prensa escrita. Universidad de Cádiz, 2000.

Lewandowska-Tomaszczyk, Barbara. Podstawy językoznawstwa korpusowego. Wydawnictwo Uniwersytetu Łódzkiego, 2005.

Nadal Palazón, Juan. «Verdades a medias: la nominalización deverbal en los titulares periodísticos». Comunicación y sociedad, vol. 9, 2008, págs. 175-190.

Nadal Palazón, Juan. «Rasgos formales de los titulares periodísticos: notas sobre diez diarios del ámbito hispánico.» Acta Poetica, vol. 33/1, 2012, págs. 173-195.

Oliva Marañón, Carlos. «Análisis léxico de los titulares de prensa en Internet». Un nuevo léxico en la red, edición a cargo de Fernando Vilches Vivancos, Dykinson, 2011, págs. 53-69.

Wolański, Adam. Edycja tekstów. Praktyczny poradnik. Wydawnictwo Naukowe PWN, 2008.

\section{CORPUS I}

marca.com, as.com, mundodeportivo.com (md.com), sport.es, sport.pl

Subcorpus I (fechas de acceso varias entre 2.02.2018 y 13.05.2018)

Subcorpus II (fechas de acceso varias entre 1.10.2020 y 20.12.2020)

\section{CORPUS II}

prensa digital (fecha de acceso entre paréntesis)

as.com [25.11.2020]

elmundo.es [30.04.2014]

elmundo.es [01.05.2014]

marca.com [04.05.2014]

marca.com [17.11.2015]

marca.com [25.11.2020]

es.yahoo.com [01.05.2014]

wp.pl [17.11.2015]

wp.pl [25.11.2020]

rtve.es [30.04.2014]

rtve.es [16 11.2015] 


\title{
RECURSOS LÉXICO-PRAGMÁTICOS \\ EN LOS TITULARES DE LA PRENSA DEPORTIVA DIGITAL
}

\begin{abstract}
Resumen
El presente texto constituye un análisis léxico formal y semántico-pragmático de los titulares digitales censados en los más grandes periódicos deportivos madirleños y catalanes. Con este fin se ha elaborado un corpus temático monolingüe, manualmente anotado, y los ejemplos censados en un portal digital polaco funcionan en calidad de tertium comparationis. Entre los medios digitales madrileños y catalanes se observan notables diferencias en cuanto a la presentación de los acontecimientos deportivos protagonizados por clubes pertenecientes a campos rivales. En cambio, el medio polaco adopta una postura objetiva aportando, en función de las circunstancias, una valoración objetiva, negativa, positiva o neutra de los acontecimientos deportivos. Una de las estratagemas discursivas que se usa con frecuencia a fin de disminuir el valor de los éxitos deportivos del campo ajeno consiste en focalizar la atención del lector en los eventos y personajes del segundo plano en detrimento del mero contenido de la noticia. Este fenómeno va relacionado con un hecho muy relevante en el plano lingüístico que diferencia la prensa catalana y la madrileña: predilección por un tipo de titular concreto o recursos formales empleados con más frecuencia.
\end{abstract}

Palabras clave: prensa digital; prensa deportiva; sensacionalismo; titulares de prensa; discurso periodístico.

\section{MECHANIZMY LEKSYKALNO-PRAGMATYCZNE W NAGŁÓWKACH ELEKTRONICZNEJ PRASY SPORTOWEJ}

Streszczenie

Artykuł stanowi analizę mechanizmów leksykalno-pragmatycznych oraz zjawisk pochodnych, jakie można zaobserwować w nagłówkach elektronicznej prasy sportowej. Analiza została przeprowadzona na tematycznym korpusie 320 nagłówków zebranych na kilku portalach sportowych: madryckich, katalońskich i polskim. Ocena wydarzeń, zwłaszcza sukcesów i porażek dwóch wielkich klubów, Realu Madryt i FC Barcelony, jest często różna i subiektywna, w zależności od tego, czy opisuje je dziennik madrycki, czy barceloński. Polski portal przedstawia zdarzenia w sposób bardziej obiektywny, neutralny i zdystansowany. Jedną ze strategii dyskursywnych, wykorzystywanych częściej przez dzienniki katalońskie, jest skierowanie uwagi czytelnika na aspekty, które nie są centralną częścią wiadomości. Różnice pomiędzy prasą ze stolicy Hiszpanii a prasą katalońską wyrażają się również w formalnym i semantycznym typie nagłówka oraz w środkach językowych najczęściej w nich stosowanych.

Słowa kluczowe: prasa sportowa; prasa elektroniczna; sensacjonalizm; dyskurs dziennikarski; nagłówki prasowe. 


\section{LEXICAL AND PRAGMATIC MEASURES \\ IN SPORTS NEWS HEADLINES ONLINE}

\section{S u m m a y}

This paper investigates the headlines in a sports news comparable corpus from a lexical, semantic and pragmatic point of view. The corpus contains articles taken from the leading online sports newspapers from Madrid and Barcelona. A Polish sports website has been used as tertium comparationis. A detailed corpus-based analysis has led to some important conclusions. First of all, we have noticed a significant difference between the Catalan and Madrilenian headlines in the way featured events involving two main rival football clubs, Real Madrid and FC Barcelona, are presented and assessed. Unlike its Spanish counterparts, the Polish website seems to take a more objective and neutral stance on the same facts. A frequently used strategy, in particular by the Catalan newspaper, consists of focusing the readers' attention on less relevant characters and events. This tendency may be related to other features, such as a marked preference for a semantic and formal type of headline on both the Madrilenian and Catalan websites, and certain formal and lexical measures which they adopt more often.

Key words: sports press; online press; sensationalism; journalistic discourse; news headlines. 